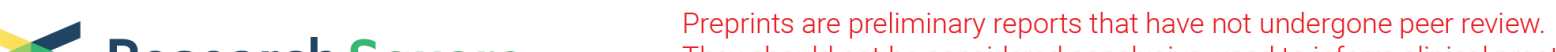 Research Square They should not be considered conclusive, used to inform clinical practice, or referenced by the media as validated information.
}

\section{Significance of Predicted Future Liver Remnant Volume on Liver Failure Risk after Major Hepatectomy: A Case Matched Comparative Study}

\section{R Piccus}

University of Birmingham

K Joshi

Queen Elizabeth Hospital Birmingham

J Hodson

Queen Elizabeth Hospital Birmingham

D Bartlett

Queen Elizabeth Hospital Birmingham

N Chatzizacharias

Queen Elizabeth Hospital Birmingham

B Dasari

Queen Elizabeth Hospital Birmingham

$\mathrm{J}$ Isaac

Queen Elizabeth Hospital Birmingham

R Marudanayagam

Queen Elizabeth Hospital Birmingham

DF Mirza

Queen Elizabeth Hospital Birmingham

JK Roberts

Queen Elizabeth Hospital Birmingham

RP Sutcliffe ( $\square$ robert.sutcliffe@uhb.nhs.uk)

Queen Elizabeth Hospital Birmingham

\section{Research Article}

Keywords: Hepatectomy, post-hepatectomy liver failure, volumetric analysis

Posted Date: February 9th, 2022

DOI: https://doi.org/10.21203/rs.3.rs-1241124/v1 
License: (c) (i) This work is licensed under a Creative Commons Attribution 4.0 International License. Read Full License 


\section{Abstract}

Introduction

Future liver remnant volume (FLRV), a risk factor for liver failure (PHLF) after major hepatectomy (MH), is not routinely measured. This study aimed to evaluate the association between FLRV and PHLF.

Patients and methods

All patients undergoing $\mathrm{MH}$ (4+ segments) between 2011-2018 were identified from a prospectively maintained single-centre database. Perioperative data were collected for patients with PHLF, who were matched (1:2) with non-PHLF controls. FLRV and FLRV $\%$ (i.e. \% of total liver volume) were calculated retrospectively from preoperative CT scans using Synapse-3D software, and compared between the PHLF and matched control groups.

Results

Of 711 patients undergoing $\mathrm{MH}, \mathrm{PHLF}$ occurred in 27 (3.8\%), of whom 24 had preoperative CT scans available. These patients were matched to 48 non-PHLF controls, $98 \%$ of whom were classified as being at high risk of PHLF on preoperative risk scoring. FLRV $\%$ was significantly lower in the PHLF group, compared to matched controls (median: 28.7 vs. $35.2 \%, p=0.010$ ), with $\mathrm{FLRV}_{\%}<30 \%$ in $58 \%$ and $29 \%$ of patients, respectively. Assessment of the ability of FLRV $\%$ to differentiate between PHLF and matched controls returned an area under the ROC curve of 0.69 , and an optimal cut-off value of $\mathrm{FLRV}_{\%}<31.5 \%$, which yielded $79 \%$ sensitivity and $67 \%$ specificity.

Conclusions

$\mathrm{FLRV}_{\%}$ is significantly predictive of PHLF after $\mathrm{MH}$, with over half of patients with PHLF having FLRV $\mathrm{F}_{\%}$ $30 \%$. In light of this, we propose that all patients should undergo risk stratification prior to $\mathrm{MH}$, with the high risk patients additionally being assessed with CT volumetry.

\section{Introduction}

Post-hepatectomy liver failure (PHLF) is an uncommon but potentially fatal complication after major hepatectomy ${ }^{1,2}$. The risk factors for PHLF are well established, and include patient demographics and the presence/severity of underlying liver disease ${ }^{3-5}$. The importance of an adequate remnant liver volume has been recognised for several decades ${ }^{6}$, and a minimum threshold future liver remnant volume (FLRV) of $20-25 \%$ of the total liver volume (TLV) had been proposed, based on historical studies of patients with normal livers ${ }^{7-9}$. Although liver volumetric software programs are available, preoperative volumetry is not universally undertaken prior to major hepatectomy, due to high cost. Whilst patients who are being considered for extended hepatectomy often undergo formal volumetric analysis, the adequacy of the FLRV before right hemihepatectomy is frequently determined by subjective assessment of the FLRV on 
preoperative imaging, due to a perceived low risk of PHLF in this group. Although the average left liver lobe volume is approximately $40 \%$ of TLV, there is a wide range of values $(18-51 \%)^{10,11}$. A significant proportion of patients have a left lobe volume less than $30 \%$ of $\operatorname{TLV}^{12,13}$, and the incidence of PHLF after right hemihepatectomy may be as high as $5 \%$, based on a study of healthy live liver donors ${ }^{14}$. The primary aim of this study was to evaluate association between volumetric analysis and PHLF after major hepatectomy.

\section{Patients And Methods}

Case selection

All patients who underwent a major hepatectomy (defined as resection of four or more segments) in a single UK tertiary hepatobiliary unit between January 2011 and December 2018 were retrospectively identified from a prospectively maintained database. Exclusion criteria were patients who underwent twostage hepatectomy (including ALPPS) or preoperative portal vein embolization $(\mathrm{N}=76)$. The group of patients developing PHLF were then identified from the database, with severity of PHLF being classified according to the ISGLS criteria ${ }^{15}$. Patients with PHLF were then matched 1:2 to patients without PHLF. Matching was performed using an ad hoc approach, with patients being matched on age, gender, type of resection (right, extended right, or extended left hemihepatectomy), indication for surgery, and preoperative chemotherapy status. Patients were exact-matched on all factors, with the exception of age, where the control case with the closest age to each PHLF case was used. In addition, it was not possible to find matches for the indication for surgery for three PHLF patients; hence this factor was disregarded when matching these cases, in order to prevent exclusions. For the matched cohorts, data for additional demographics, as well as peri- and postoperative factors were collected, with the expected risk of PHLF being quantified using the preoperative PHLF risk score ${ }^{16}$.

CT volumetric analysis

During the study period, for patients undergoing extended (left or right) hepatectomy, CT volumetric analysis was performed selectively in cases where it was felt to be warranted, based on the surgeon's subjective assessment of the future liver remnant (FLR). Preoperative CT volumetric analysis was not routinely undertaken in patients undergoing right hemihepatectomy. As such, for patients in the PHLF and matched control groups, liver volumetric analysis was carried out retrospectively from preoperative CT scans, using Fujifilm Synapse imaging software (Fujifilm, Japan). From this, the right (RLV) and left liver lobe volumes (LLV) were calculated, both as absolute values and as a proportion of TLV (e.g. RLV $\%=$ $100 *[R L V / T L V])$. The volume to be resected was then calculated, and used to estimate FLRV. Again, this was assessed as both an absolute value, and as a percentage of TLV (FLRV $\%=100 *[F L R V / T L V])$. The $\mathrm{FLRV}_{\%}$ was deemed to be "inadequate" if $<30 \%$, "borderline" if $30-39 \%$ and "adequate" if $\geq 40 \%$. 
Statistical methods

Initially, the control patients included in the matched cohort were compared to those not selected by the matching procedure, in order to assess how the matched cohort compared to the population as a whole. Within the matched cohort, comparisons were then made between PHLF and control patients, to test whether these groups were comparable at baseline. In each case, ordinal or continuous variables were compared using Mann-Whitney $U$ tests and summarised using the mean \pm standard deviation (SD) where approximately normally distributed, with the median and interquartile range (IQR) reported otherwise. Nominal variables were analysed using Fisher's exact tests. The ability of CT volumetry data to differentiate between the PHLF and matched control groups was then assessed using receiver operating characteristic (ROC) curves, and quantified using the area under the curve (AUROC). A binary logistic regression model was also produced, with the $F L R V_{\%}$ as a continuous covariate, in order to visualise the relationship. The optimum cut-off value of $\mathrm{FLRV}_{\%}$ for the discrimination between the PHLF and matched control groups was then estimated, based on the value with the highest Youden's $\mathrm{J}$ statistic. All analyses were performed using IBM SPSS 24 (IBM Corp. Armonk, NY), with $p<0.05$ deemed to be indicative of statistical significance throughout.

\section{Results}

Overall cohort characteristics

A total of 711 patients underwent major hepatectomy during the study period, including 476 right hemihepatectomies $(\mathrm{RH})$ and 235 extended hemihepatectomies ( $\mathrm{EH}$; comprising 152 extended right, and 83 extended left). Of the entire cohort, 27 patients (3.8\%) developed PHLF, with 5, 11 and 11 of grade A, B and C, respectively. The incidence of PHLF after RH and EH was 12/476 (2.5\%) and 15/235 (6.4\%), respectively. There were six PHLF-related deaths, including two deaths after $\mathrm{RH}$.

Liver volumetry in patients developing PHLF

Preoperative CT scans were unavailable for 3/27 (11\%) patients with PHLF; hence retrospective CT liver volumetric analysis could only be performed in the remaining 24 cases. The demographics of this group are reported in Table 1, with liver volumetry reported in Table 2. These patients had a median TLV of 1707 $\mathrm{ml}$ (IQR: 1361-2154), and median left and right lobe volumes of $656 \mathrm{ml}$ (IQR: 499-848) and $932 \mathrm{ml}$ (IQR: 839-1312), respectively. The left lobe volume was a median of 37.1\% (IQR: 31.5-43.6) of TLV, and three patients (25\%) who developed PHLF after RH had a left lobe volume of $<30 \%$ of TLV. The median FLRV\% in patients undergoing $\mathrm{RH}$ (30.6\%, IQR: 28.2-31.2) was significantly higher than in those undergoing $\mathrm{EH}$ (26.5\%, IQR: 22.6-29.3; $\mathrm{p}=0.014$ ), with $\mathrm{FLRV}_{\%}$ being $<30 \%$ in $4 / 11(36 \%)$ and $10 / 13(77 \%)$ patients who developed PHLF after RH and EH, respectively. Three patients developed PHLF despite having FLRV $\geq 40 \%$, including two patients who developed grade B PHLF after right hemihepatectomy and had 
radiological drainage of intra-abdominal collections. The third patient developed severe venous congestion of the liver remnant following an extended left hemihepatectomy with significant intraoperative blood loss, and subsequently developed multi-organ failure and died on the third postoperative day.

Selection of matched controls

The 27 patients with PHLF were then matched in a 1:2 ratio to 54 control patients without PHLF. After excluding those for whom preoperative CT scans were unavailable, 24 patients with PHLF and 48 patients without PHLF were included in subsequent analysis. Comparisons between the matched controls and 630 unmatched controls found those included in the matched analysis to be significantly more likely to be male ( $75 \%$ vs. $54 \%, p=0.004)$, to have cholangiocarcinoma ( $35 \%$ vs. $8 \%, p<0.001)$ and to have undergone extended right hemihepatectomy ( $46 \%$ vs. $18 \%, p<0.001)$ than the remainder of the non-PHLF group (Table 1). As such, the matched controls were identified as being a biased sample of the cohort as a whole with very high risk of PHLF, as would be expected given the matching procedure; the median preoperative risk score for matched controls was 10.3 (IQR: 9.0-10.5), and 98\% (47/48) had risk scores above the proposed high-risk threshold of $>5.5^{16}$.

Comparisons between patients developing PHLF and matched controls

Comparisons between the PHLF and matched control groups found these to have similar baseline characteristics, including similar PHLF risk scores (median: 10.5 vs. $10.3, p=0.247$ ); $38 \%$ of patients in each cohort received chemotherapy prior to hepatectomy (Table 1). Analysis of liver volumetry (Table 2) found the TLV to be similar in the PHLF and matched control groups (median: 1707 vs. $1595 \mathrm{ml}$, $p=0.685$ ). However, the left liver volume was a significantly smaller percentage of TLV in those developing PHLF, compared to matched controls (median: 37.1 vs. $41.6 \%, p=0.025$ ).

The average $\mathrm{FLRV}_{\%}$ was significantly lowerin the PHLF group compared to matched controls (median 28.7 vs. $35.2 \%$; $p=0.010$ ), with $58 \%$ vs. $29 \%$ having $F_{R R V}<30 \%$. The associated AUROC for the differentiation between the PHLF and matched control groups was 0.69 (95\% Cl: 0.56-0.82), with binary logistic regression returning an odds ratio of 0.92 (95\% Cl: 0.86-0.99) per unit increase in $\mathrm{FLRV}_{\%}$ (Figure 1). Based on the Youden's $J$ statistic, the optimum cut-off value of $F L R V_{\%}$ for discriminating between the PHLF and matched control groups was 31.5\%. Of those with $\mathrm{FLRV}_{\%}<31.5 \%, 19 / 35$ (54\%) were in the PHLF group, compared to $5 / 37$ (14\%) of those with $\mathrm{FLRV}_{\%} \geq 31.5 \%$, yielding $79 \%$ sensitivity and $67 \%$ specificity.

Perioperative factors and postoperative outcomes

The proportions of patients who underwent a concomitant vascular resection or had inflow occlusion were similar in the PHLF and matched control groups (Table 1). The incidence of postoperative bile leak, intra-abdominal sepsis and portal vein thrombosis were also similar between groups. However, patients developing PHLF were significantly more likely to receive a perioperative blood transfusion (48\% vs. $15 \%$; 
$p=0.005)$ and to require radiological drainage of intra-abdominal collections $(46 \%$ vs. $10 \% ; p=0.002)$ than matched controls.

\section{Discussion}

The importance of the adequacy of the future liver remnant volume to minimize the risk of PHLF after major hepatectomy is well established ${ }^{17}$. However, liver volumetric analysis is not routinely performed prior to major hepatectomy in many centres, due to cost and/or limited resources. The primary aim of this study was to assess the relationship between liver volumetric analysis and PHLF. This would be useful in determining whether routine utilisation of preoperative volumetry analysis in treatment planning could have the potential to reduce the incidence of PHLF.

The most notable finding of this study is that more than half of patients who developed PHLF in this series had an "inadequate" FLRV $\%$ of $<30 \%$, including one third of patients who developed PHLF after RH. All 14 patients with FLRV $_{\%}<30 \%$ who developed PHLF were classified as high-risk based on preoperative risk scoring (scores >5.5). As such, if preoperative volumetric analysis had been undertaken for all highrisk patients in this series, then treatment plans could have been modified in these patients, in order to minimise the risk of PHLF. For example, these patients could have been referred for preoperative portal/hepatic vein embolization to induce hypertrophy of the liver remnant, or may have been considered for non-surgical therapies. Because PHLF has no effective treatment, management is aimed at multiorgan support and is associated with significant morbidity, mortality and healthcare costs. ${ }^{18}$ Prevention of PHLF is therefore essential, and based on data from this study, preoperative volumetric analysis can be justified in high-risk patients prior to major hepatectomy, including right hemihepatectomy.

Whilst the majority of patients developing PHLF had an "inadequate" FLRV $\%$ PHLF also developed in

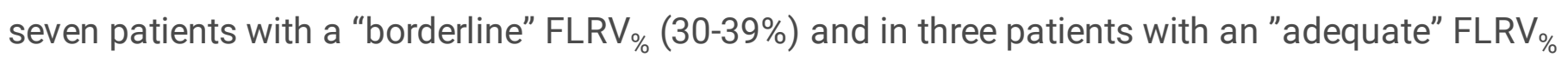
( $\left.{ }^{3} 40 \%\right)$. This may be, in part, due to the threshold used for "inadequate" FLRV $\%$, with the optimum cut-off in our analysis being higher, at $31.5 \%$. In addition, in patients with borderline or adequate FLR, the mechanism of PHLF is likely to be multifactorial, including impaired global liver function and/or postoperative complications (e.g. haemorrhage, vascular thrombosis or sepsis). A significant proportion of patients undergoing major hepatectomy have parenchymal liver injury (e.g. chemotherapy induced damage, biliary obstruction or steatosis/steatohepatitis) that may predispose patients to impaired postoperative liver function and/or PHLF. Although reversible causes such as biliary obstruction must be corrected prior to surgery, the impact of irreversible causes on global liver function may be quantified preoperatively. The $99^{\mathrm{m}}$ Technetium-Mebrofinate SPECT-CT scan is an emerging modality that provides anatomical and functional assessment of liver function, but is not yet widely available. ${ }^{19}$ Meticulous surgical technique and prompt diagnosis and treatment of postoperative complications are essential in order to minimize the impact on liver function in patients with borderline $F L R V_{\%}$. 
In addition to the 14 patients with PHLF with "inadequate" FLRV on preoperative liver volumetry, there were a further 14 matched controls who did not develop PHLF, despite having FLRV $\%<30 \%$. These patients comprised $29 \%$ of the matched control cohort, although this is likely a considerable overestimate of the rate of "inadequate" FLRV ${ }_{\%}$ in the non-PHLF population as a whole, since the matching procedure selected a highly biased and very high-risk control cohort. Despite this, these patients highlight that use of preoperative liver volumetry in isolation to plan treatment may result in under-treatment in some patients who would not have gone on to develop PHLF. As such, preoperative liver volumetry needs to be utilised alongside other information during the decision-making process.

This study has several limitations. None of the patients with PHLF in our series had underlying cirrhosis, and this is a reflection of the small number of cirrhotic patients who undergo major hepatectomy in our unit. Therefore, the results of this study cannot be applied to this subgroup, and further study of cirrhotic patients would be required. Whilst matching PHLF patients to controls constituted a strength of the study, by negating the effect of potentially confounding factors on the comparisons between groups, it also represented a limitation. The process of matching control patients to those with PHLF resulted in the selection of higher risk control patients, with a greater proportion of males, patients with cholangiocarcinoma, and those undergoing extended surgeries, compared to the controls not included in the study. As such, the cases included in the study are a biased subset of the cohort as a whole. Consequently, if the associations considered in the analysis vary with PHLF risk, then the findings may not be applicable to the cohort of patients undergoing hemihepatectomy as a whole.

In conclusion, the incidence of post-hepatectomy liver failure could potentially be reduced by performing liver volumetric analysis prior to major hepatectomy in high-risk patients. Although the extent of resection is an important factor, a large proportion of cases of PHLF in this series occurred after right hemihepatectomy.

\section{Declarations}

Competing Interests

There are no competing interests to declare.

Ethical approval and consent to participate

All methods were carried out in accordance with relevant guidelines, in accordance with the Helsinki Declaration. Due to the retrospective nature of this study, ethical approval and consent was waived by the institutional audit committee at Queen Elizabeth Hospital, Birmingham and clinical audit management team with CARMS number 16463.

Consent for publication

The authors hereby give Biomedcentra/ the full rights to publish this manuscript in BMC Surgery 
Availability of data and materials

The datasets generated during and analyzed during the current study are not publicly available, as this is not a mandatory requirement by our institutional audit committee, but they are available from the corresponding author on reasonable request.

Funding

This study did not receive any external funding

Authors' Contributions

$\mathrm{RP}, \mathrm{JH}, \mathrm{KH}$ and RS contributed to the main manuscript. JH prepared the figures. RP and $\mathrm{KJ}$ analysed the data. RP, KJ, RS, KR, DM, RM, JI, BD, NC and DB collected data. Statistical analysis was by JH and RS. All authors reviewed the manuscript.

Acknowledgements

None

\section{References}

1. Schrekenbach T, Liese J, Bechstein WO, Moench C. Posthepatectomy liver failure. Dig Surg 2012, 29, 79-85.

2. Gilg S, Sandstrom P, Rizell M, Lindell G, Ardnor B, Stromberg C, Isaksson B. The impact of posthepatectomy liver failure on mortality: a population based study. Scandanavian Journal of Gastroenterology 2018, 53, 1335-39.

3. Rahnemai-Azar AA, Cloyd JM, Weber SM, Dillhoff M, Schmidt C, Winslow ER, Pawlik TM. Update on liver failure following hepatic resection: strategies for prediction and avoidance of post-operative liver insufficiency. J Clin Transl Hepatol 2018, 6, 97-104

4. Wiggans MG, Lordan JT, Shahtahmassebi G, Aroori S, Bowles MJ, Stell DA. The interaction between diabetes, body mass index, hepatic steatosis, and risk of liver resection: insulin dependent diabetes is the greatest risk for major complications. HPB Surg 2014, 2014:586159

5. Soreide JA, Deshpande R. Post hepatectomy liver failure (PHLF) - recent advances in prevention and clinical management. Eur J Surg Oncol 2021, 47, 216-224.

6. Jarnagin WR, Gonen M, Fong Y, DeMatteo RP, Ben-Porat L, Little S, Corvera C, Weber S, Blumgart LH. Improvement in perioperative outcome after hepatic resection: analysis of 1,803 consecutive cases over the past decade. Ann Surg 2002, 236, 397-406

7. Khan AS, Garcia-Aroz S, Ansari MA, Atiq SM, Senter-Zapata M, Fowler K, Doyle MB, Chapman WC. Assessment and optimization of liver volume before major hepatic resection: current guidelines and a narrative review. Int J Surg 2018, 52, 74-81 
8. Kishi Y, Abdalla EK, Chun YS, Zorzi D, Madoff DC, Wallace MJ, Curley SA, Vauthey J-N. Three hundred and one consecutive extended right hemihepatectomies: evaluation of outcome based on systematic liver volumetry. Ann Surg 2009, 250, 540-48

9. Shoup M, Gonen M, D'Angelica M, Farnagin WR, DeMatteo RP, Schwarts LH, Tuorto S, Blumgart LH, Fong Y. Volumetric analysis predicts hepatic dysfunction in pateints undergoing major liver resection. Journal of Gastrointestinal Surgery 2003, 7, 325-330

10. Tongyoo A, Pomfret EA, Pomposelli JJ. Accurate estimation of living donor right hemi-liver volume from portal vein diameter measurement and standard liver volume calculation. American Journal of Transplantation 2012, 12, 1229-39.

11. Kim YH, Park UJ, Chung HS, Hong SH, Park CS, Choi JH, Choi HJ, Jung J-Y, Chae MS. Recovery of the psoas muscle index in living donors after a right lobe hepatectomy for liver transplantation: a singlecenter experience. Transplantation Proceedings 2019, 51, 1853-1860.

12. Abdalla EK, Denys A, Chevalier $P$, Nemr RA, Vauthey JN. Total and segmental liver volume variations: implications for liver surgery. Surgery 2004;135:404-410. doi: 10.1016/j.surg.2003.08.024

13. Leelaudomlipi S, Sugawara Y, Kaneko J, Matsui Y, Ohkubo T, Makuuchi M. Volumetric analysis of liver segments in 155 living donors. Liver Transplant 2002, 6, 612-14

14. Rossler F, Sapisochin G, Song G, Lin Y-H, Simpson MA, Hasegawa K, Laurenzi A, Cabus SS, Nunez MI, Gatti A, Beltrame MC, Slankamenac K, Greig PD, Lee S-G, Chen C-L, Grant DR, Pomfret EA, Kokudo N, Cherqui D, Olthoff KM, Shaked A, Garcia-Valdecasas JC, Lerut J, Troisi RI, De Santibanes M, Petrowsky H, Puhan MA, Clavien P-A. Defining benchmarks for major liver surgery: a multicenter analysis of 5202 living liver donors. Ann Surg 2016, 264, 492-500.

15. Rabhari NN, Garden J, Padbury R, Brooke-Smith M, Crawford M, Adam R, Koch M, Makuuchi M, Dematteo RP, Christophi C, Banting S, Usatoff V, Nagino M, Maddern G, Hugh TJ, Vauthey J-N, Greig P, Rees M, Yokoyama Y, Fan ST, Nimura Y, Figueras J, Capussotti L, Buchler MR, Weitz J. Posthepatectomy liver failure: a definition and grading by the international study group of liver surgery (ISGLS). Surgery 2011, 149, 713-24.

16. Dasari BVM, Hodson J, Roberts KJ, Sutcliffe RP, Marudanayagam R, Mirza DF, Isaac J, Muiesan P. Developing and validating a pre-operative risk score to predict post-hepatectomy liver failure. HPB (Oxford) 2019, 21, 539-46

17. Ray S, Mehta NN, Golhar A, Nundy S. Posthepatectomy liver failure - a comprehensive review of current concepts and controversies. Ann Med Surg (Lond) 2018, 34, 4-10

18. Yadav K, Shrikhande S, Goel M. Post hepatectomy liver failure: concept of management. J Gastrointest Canc 2014, 45, 405-13.

19. Dasari BMV, Wilson M, Pufal K, Kadam P, Hodson J, Roberts KJ, Chatzizacharias N, Marudanayagam R, Gadvi R, Sutcliffe RP, Mirza DF, Muiesan P, Isaac J. Variations between the anatomical and functional distribution, based on $99^{\mathrm{m}}$ technetium-mebrofinate SPECT-CT scan, in patients at risk of post hepatectomy liver failure. HPB (Oxford) 2021, S1365-182; (21)0014 


\section{Tables}

Table 1. Cohort characteristics 
Matched

Age (years)

Gender (\% male)

$\operatorname{BMI}\left(\mathrm{kg} / \mathrm{m}^{2}\right)$

Liver disease

None

Post-chemotherapy

Cholestasis

Steatosis/steatohepatitis

Fibrosis/cirrhosis

Myocardial infarction

Diabetes mellitus

Charlson comorbidity index

Indication for surgery

Colorectal metastases

Cholangiocarcinoma

Hepatocellular carcinoma

Others

Preoperative

chemotherapy ${ }^{d}$

PHLF risk score ${ }^{\mathbf{e}}$

$24 \quad 10.5$

(9.3-

11.0)
24

$5(21 \%)$

$4(17 \%)$

$2(8 \%)$

$0(0 \%)$

$243(13 \%)$

$245(21 \%)$

$24 \quad 9(5-9)$

24
$13(54 \%)$
29

(60\%)

$8(17 \%)$

$8(17 \%)$

$2(4 \%)$

$1(2 \%)$
$11(46 \%)$

$8(33 \%)$

$0(0 \%)$

$5(21 \%)$
25

(52\%)

17

(35\%)

\section{PHLF Control}

N Statistic N Statistic

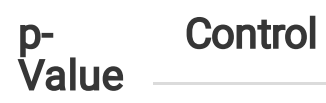

N Statistic b

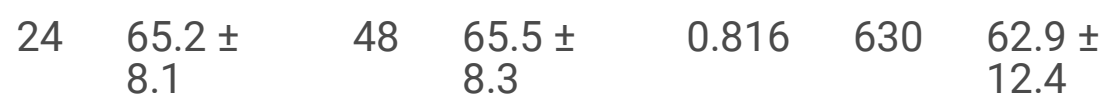

$\begin{array}{llll}24 & 18(75 \%) & 48 & 36 \\ & & (75 \%)\end{array}$

$24 \quad 26.8 \pm$

4.6

$47 \quad 28.2 \pm$

4.3

N/A $\quad 630 \quad 338$

(54\%)

$0.193 \quad 560 \quad 28.0 \pm$

4.9
Value

0.267

0.004

0.856
48

0.896
$48 \quad 2(4 \%)$

$48 \quad 4(8 \%)$

$48 \quad 8(5-9)$

48

0.663

630

$<0.001$
407

$(65 \%)$

$53(8 \%)$

$47(7 \%)$

123

(20\%)

\begin{tabular}{ll}
$0(0 \%)$ & $47(7 \%)$ \\
\hline $6(13 \%)$ & 123 \\
& $(20 \%)$
\end{tabular}

Type of surgery

24

48

N/A

630

$<0.001$

Right hemihepatectomy

$11(46 \%) \quad 22$




\begin{tabular}{|c|c|c|c|c|c|c|c|c|}
\hline $\begin{array}{l}\text { Extended right } \\
\text { hemihepatectomy }\end{array}$ & & $11(46 \%)$ & & $\begin{array}{l}22 \\
(46 \%)\end{array}$ & & & $\begin{array}{l}113 \\
(18 \%)\end{array}$ & \\
\hline $\begin{array}{l}\text { Extended left } \\
\text { hemihepatectomy }\end{array}$ & & $2(8 \%)$ & & $4(8 \%)$ & & & $\begin{array}{l}77 \\
(12 \%)\end{array}$ & \\
\hline Vascular resection & 24 & $3(13 \%)$ & 48 & $9(19 \%)$ & 0.739 & - & - & - \\
\hline Inflow occlusion & 24 & $7(29 \%)$ & 48 & $\begin{array}{l}16 \\
(33 \%)\end{array}$ & 0.794 & - & - & - \\
\hline $\begin{array}{l}\text { Perioperative blood } \\
\text { transfusion }\end{array}$ & 21 & & 48 & & $\begin{array}{l}0.005 \\
c\end{array}$ & 624 & & $\begin{array}{l}0.432 \\
c\end{array}$ \\
\hline None & & $11(52 \%)$ & & $\begin{array}{l}41 \\
(85 \%)\end{array}$ & & & $\begin{array}{l}503 \\
(81 \%)\end{array}$ & \\
\hline $1-2$ units & & $7(33 \%)$ & & $4(8 \%)$ & & & $\begin{array}{l}76 \\
(12 \%)\end{array}$ & \\
\hline$>2$ units & & $3(14 \%)$ & & $3(6 \%)$ & & & $45(7 \%)$ & \\
\hline
\end{tabular}

Postoperative complications

Bile leak/intra-abdominal sepsis

Portal vein thrombosis

Radiological drainage of $24 \quad 2(8 \%) \quad 48 \quad 7(15 \%) \quad 0.708$ $24 \quad 1(4 \%) \quad 48 \quad 0(0 \%)$ $0.333-$ fluid collections $24 \quad 11(46 \%) \quad 48 \quad 5(10 \%) \quad 0.002 \quad-$

Data were only collected for selected variables in the unmatched control cohort. Ordinal and continuous variables are reported as mean \pm standard deviation, or as median (interquartile range), with $p$-values from Mann-Whitney $U$ tests. Nominal variables are reported as $N$ (column \%), with $p$-values from Fisher's exact tests, unless stated otherwise. Bold p-values are significant at $p<0.05$. N/A = not applicable, as patients were exact-matched on the stated factor. ${ }^{a} p$-Values comparing the PHLF vs. control groups in the matched cohort. ${ }^{b} p$-Values comparing the control groups in the matched vs. unmatched cohorts. ${ }^{c} p$ Value from Mann-Whitney $U$ test, as the factor is ordinal. ${ }^{d}$ With Oxaliplatin or Irinotecan. ${ }^{\mathbf{e}}$ Reference 16

\section{Table 2. Volumetric analysis}




\begin{tabular}{|c|c|c|c|c|}
\hline & $\begin{array}{l}\text { PHLF } \\
(\mathrm{N}=24)\end{array}$ & $\begin{array}{l}\text { Matched Control } \\
(\mathrm{N}=48)\end{array}$ & $\begin{array}{l}\text { AUROC } \\
(95 \% \mathrm{Cl})^{a}\end{array}$ & $\begin{array}{l}\text { p- } \\
\text { Value }\end{array}$ \\
\hline Left liver volume (ml) & $656(499-848)$ & $677(543-822)$ & $0.55(0.40-0.70)^{b}$ & 0.488 \\
\hline Left liver volume (\%) & $37.1(31.5-43.6)$ & $41.6(36.7-47.0)$ & $0.66(0.52-0.80)^{b, c}$ & $0.025^{c}$ \\
\hline Right liver volume (ml) & $932(839-1312)$ & 911 (764-1179) & $0.60(0.47-0.74)$ & 0.152 \\
\hline Right liver volume (\%) & $62.9(56.4-68.5)$ & $58.4(53.1-63.4)$ & $0.66(0.52-0.80)^{c}$ & $0.025^{c}$ \\
\hline Total liver volume (ml) & $1707(1361-2154)$ & $1595(1393-1942)$ & $0.53(0.38-0.68)$ & 0.685 \\
\hline Volume resected $(\mathrm{ml})$ & 1175 (964-1442) & $1079(864-1265)$ & $0.60(0.46-0.74)$ & 0.164 \\
\hline FLRV (ml) & $486(399-590)$ & $546(439-687)$ & $0.61(0.47-0.76)^{b}$ & 0.116 \\
\hline $\mathrm{FLRV}_{\%}(\%)$ & $28.7(26.1-31.2)$ & $35.2(27.9-39.4)$ & $0.69(0.56-0.82)^{b}$ & 0.010 \\
\hline$<30 \%$ (inadequate) & $14(58 \%)$ & $14(29 \%)$ & & \\
\hline 30-39\% (borderline) & $7(29 \%)$ & $23(48 \%)$ & & \\
\hline${ }^{3} 40 \%$ (adequate) & $3(13 \%)$ & $11(23 \%)$ & & \\
\hline
\end{tabular}

Data are reported as median (interquartile range), or as $\mathrm{N}(\%)$, with p-values from Mann-Whitney $\mathrm{U}$ tests. Bold $p$-values are significant at $p<0.05 .{ }^{a}$ Area under the receiver operating characteristic curve (AUROC); AUROCs $>0.5$ indicate factors where values were higher in those with PHLF, unless stated otherwise. b Values were lower in those with PHLF. ${ }^{c}$ Since the left liver volume as a percentage of the total is the inverse of the right, the AUROCs and $p$-values are identical for both factors.

\section{Figures}




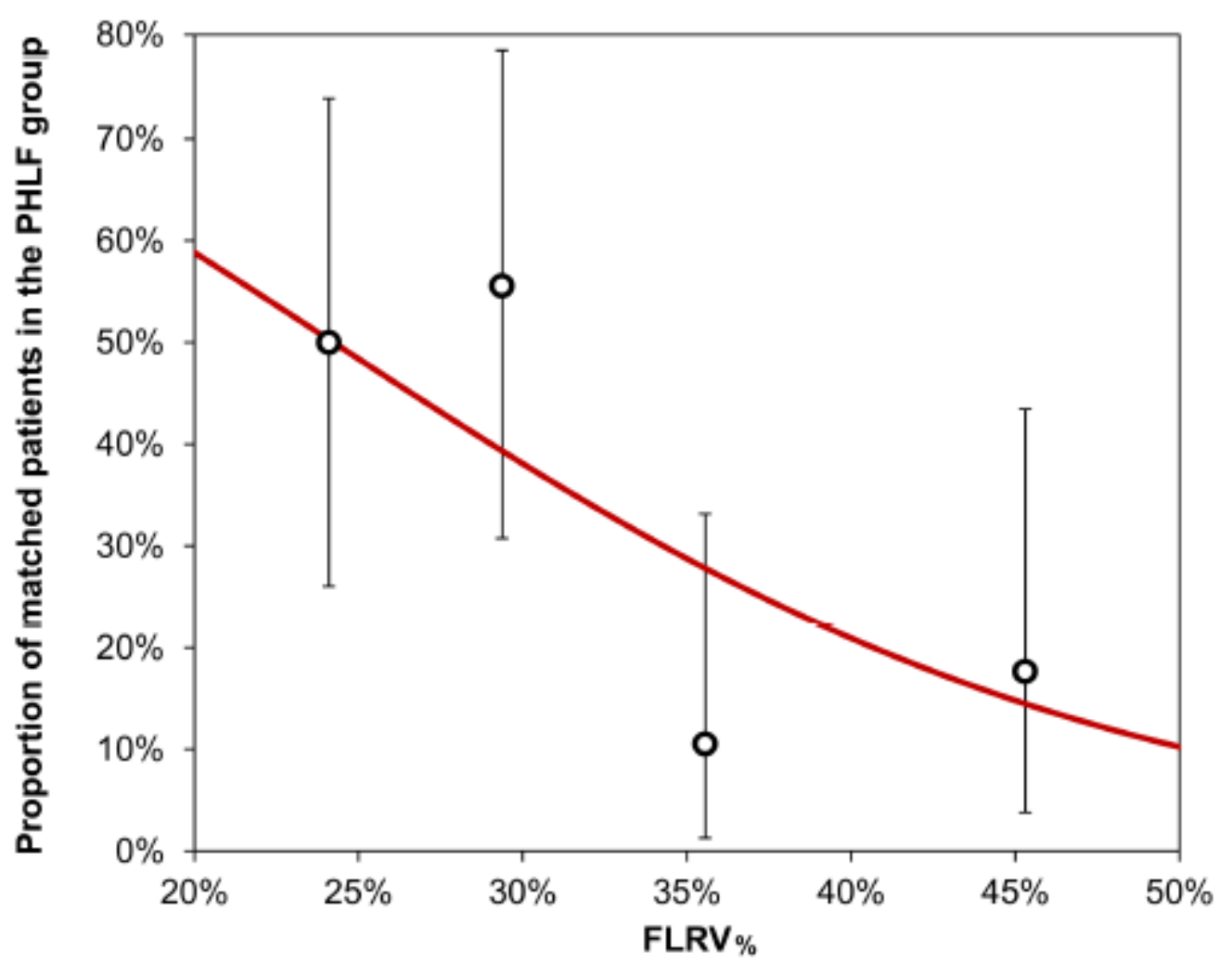

Figure 1

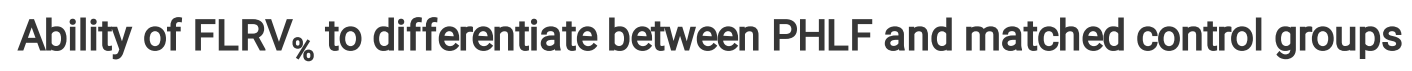

The trend line is from a binary logistic regression model, with the FLRV $\%$ as a continuous covariate. In order to visualise the goodness of fit, points represent the proportions of patients with PHLF within groups defined by the quartiles of $\mathrm{FLRV}_{\%}$, and are plotted at the mean $\mathrm{FLRV}_{\%}$ of each group, with whiskers representing $95 \%$ confidence intervals. 